\title{
Imperceptible Printer Dot Watermarking for Binary Documents
}

\author{
J. A. Briffa, C. Culnane, H. Treharne \\ University of Surrey, Guildford, Surrey, UK
}

\begin{abstract}
In this paper we propose a new imperceptible yellow printer dot watermarking scheme for documents printed on a colour printer. The scheme takes advantage of the imperfections of the human visual system to hide thousands of yellow dots over the entire page. It takes inspiration from the methods used by laser printer manufacturers for printer identification.

The novelty of our approach is in providing an automatic embedding and detection method that can survive the distortions of printing and scanning. In order to achieve this a new moving window detection method is proposed. An error correction code is employed to handle the errors that could be present following detection.

The scheme is evaluated through embedding and detection experiments on different types of documents; including text, architectural drawings and a cartoon. Our scheme offers an embedding capacity of 26,190 bits per page. Experiments were conducted using error correction codes with rates of $\frac{1}{2}, \frac{1}{3}$ and $\frac{1}{5}$, given payloads of $13,095,8,730$, and 5,238 bits per A4 page respectively. We are able to successfully recover the watermark in all documents at a rate of $\frac{1}{2}$ and $\frac{1}{5}$, and in all document except one at $\frac{1}{3}$. Further experiments were conducted with a smaller dot size to evaluate the impact it has on our results. With the smaller dot size we were still able to recover the watermarks from all documents when using an error correction code with rate $\frac{1}{5}$.

The capacity offered by this approach far exceeds the capacity offered by existing binary watermarking schemes, which are robust to printing and scanning. The substantially larger capacity opens up a wider range of possible applications as well as the possibility of using more robust cryptographic techniques for authentication.
\end{abstract}

Keywords: W AT

\section{INTRODUCTION}

One of the earliest binary watermarking schemes that was resilient to printing and scanning was, ${ }^{1}$ published in 1998. However, a little known form of digital watermarking has been in use for a number of years. ${ }^{2}$ The technique described by the Electronic Frontier Foundation (EFF) in ${ }^{3}$ involves hiding a grid of tiny yellow dots on the page, in order to embed the identification number of the printer. The exact technique varies between different printer manufactures. The reason behind this technique appears to be for the identification of counterfeit notes and tickets. It has been shown to have success in detecting and identifying the culprits of these crimes. ${ }^{4}$ However, there is concern over the invasion of privacy that the inclusion of these identifying dots presents. ${ }^{5}$

The techniques rely on the imperceptibility of yellow dots on white paper. Generally it is a fairly crude system that repeats the same pattern many times across a page. There is no attempt to avoid areas where the watermark will be lost, for example sections of text or black boxes. Some schemes, in particular the Xerox system discussed in, ${ }^{6}$ offset the grid of dots to attempt to reduce the chances of errors. The exact start date of this technique and even the full details are shrouded in secrecy and few manufacturers have fully admitted the extent to which the system is deployed. The EFF list over 130 printers that they have currently detected employ some form of this watermarking technique. An example of a dot pattern from a Hewlett Packard (HP) printer can be seen in Figure 1. It appears as if the watermark is designed to be read by a human and a high power lens, as opposed to an automatic system that can use a standard scanner. However, this cannot be confirmed due to lack of information. Our novel scheme, adopts the principles used by the printer manufacturers, in order

Further author information: (Send correspondence to J. A. Briffa)

J. A. Briffa: E-mail: j.briffa@surrey.ac.uk 


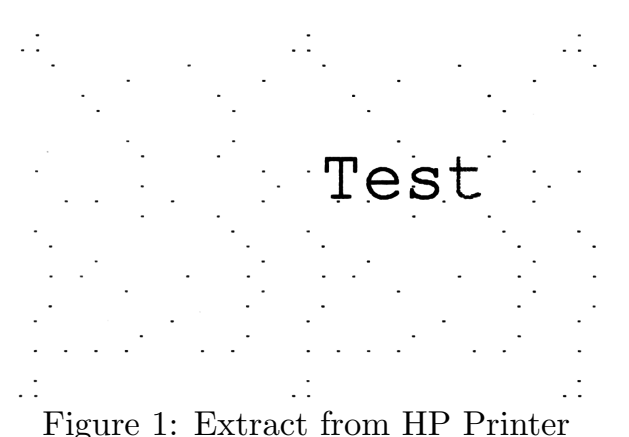

Figure 1: Extract from HP Printer

to develop a system that allows custom data to be embedded within printouts of text and line art. Our scheme's strength is its high capacity and robustness to printing and scanning.

Obviously the technique cannot be used with colour laser printers that already print identification dots, since they will conflict with each other. We recommend using an ink jet printer, since they are not known to print identification dots. Our proposed application for such a system is document authentication, with improved security options, due to the greater capacity available compared to previous binary watermarking schemes that are robust to printing and scanning, ${ }^{78}$ and. ${ }^{9}$ In our previous work,,${ }^{9}$ we achieved a maximum capacity of 115 bits per page. In this paper we will demonstrate a capacity of 26190 bits per page.

\section{PRINTER CODE IDENTIFICATION}

We analysed the printer identification dots created by an HP LaserJet 3600DN printer. There already exists a thorough analysis of the dots for Xerox printers in $^{6}$ and there is an online application that decodes these. ${ }^{3}$ The easiest way to view the dots is by shining a blue LED light on the page, although in the case of the HP we have had success at using a scanner to scan the page at 600 dpi. Figure 1 shows an extract from a scan of a page printed on the HP Laserjet 3600DN. It has been contrast enhanced in order to allow the dots to be seen in a greyscale printout. The grid structure of the dots and also the small size of the grid are clearly visible, indicating the small capacity available. This pattern is repeated across the whole page from left to right and from top to bottom. Unlike the Xerox scheme ${ }^{6}$ there is no offsetting of the grid.

We have tried printing different documents, at different times under different usernames, but always receive

the same dot output pattern. It would appear that unlike the Xerox scheme, ${ }^{6}$ the HP 3600 DN only outputs a serial number and does not include data about time, date or document.

\section{OVERVIEW OF OUR SCHEME}

The yellow dot technique has been applied to printer identification, as in, ${ }^{6}$ however, we wanted to establish if a similar scheme could be used, using an ink jet printer, to embed and detect an imperceptible watermark that could be used for document authentication. The rest of this paper discusses the techniques we have developed to achieve this as a proof-of-concept.

The aim of the technique is to provide automatic embedding and detection of the yellow dots into binary documents, in a manner robust to printing and scanning, at a resolution of $600 \mathrm{dpi}$. This requires automatic handling of geometric distortion and intelligent embedding to minimise perceptibility.

Our initial work looked at using dynamically sized grids, however, to simplify the process we have currently fixed the size of the grid. The size could be varied according to the application and paper size, but for a proof-of-concept a fixed grid is sufficient.

We fixed the size of the grid at 194 rows by 135 columns. This was calculated based on the size of a typical page of A4, allowing for margins around the page, and gaps between dots. The capacity of the grid is 26190 bits, a significant portion of this will be used for error correction so the message size will depend on the error coding utilised, details of those tested can be found in Section 6. Our capacity is over twice what can be achieved in, ${ }^{7}$ e.g., for our sample document their theoretical capacity of 3 bits per character, would provide 10347 bits. 
We investigated the use of control points to aid the identification of rows and columns. This was particularly useful when using a dynamically sized grid, however, it proved not to be robust enough. Too high an importance was placed on the control dots, without being able to provide any added protection to them. With a fixed sized grid we can remove the need for the control points.

There are a number of constants that are used during the creation of the grid and the original calculations of the grid size. The following is a list of these constant values, in pixels, along with a description.

- $C_{w}=30, C_{h}=30$ are the width and height of an individual cell

- $D_{w}=5, D_{h}=5$ are the width and height of an individual dot

- $P_{w}=4958, P_{h}=7017$ are the approximate rasterised width and height of an A4 document at 600dpi

- $G_{m}=100$ is the minimum margin permitted around the grid. This ensures that we have the space to crop the typical scanner noise caused around the edge of the image caused by the intersection of the paper and the scanner lid.

The $C_{w}$ and $C_{h}$ determine the level of rotational distortion that can be handled. The greater the value, the more rotation that can be handled and is a consequence of how we locate the grid during detection. With a gap of 30, the theoretical rotation that can be handled is approximately $1.5^{\circ}$.

We try to keep the grid the same overall size, even when varying the size of the dots. Therefore, when we experimented with a smaller dot size of just $3 \times 3$ pixels, we increased the cell width and height by 2 to 32 . We could have alternatively created different error correction codes and changed the initial size of the grid to increase the capacity. The increase in robustness to rotational distortion is insignificant.

\section{EMBEDDING}

In this section we step though the embedding process providing a detailed description of how we embed and the reasons behind the choices we have made. The example document is an A4 page of text in the Portable Network Graphics (PNG) format at $600 \mathrm{dpi}$. The data to be embedded is read in as a binary string, having previously been encoded using the appropriate error correction technique. This data is stored into a 2 dimensional array that represents where we will place the dots. If the bit is a ' 1 ' we will endeavour to place a yellow dot, if it is a zero we will not.

The dots are rendered as a number of yellow pixels with a value of 255, 255, 110 for Red, Green, and Blue respectively. This value was chosen based on the colour we detected when using the HP 3600DN. In order to minimise the perceptibility of the yellow dots it is important that they are only embedded on areas of white pixels. If the dot is placed on black or shades of grey it is particularly perceptible. We therefore iterate through the location where the dot is to be placed and only render a yellow pixel when the existing pixel is white. This can result in partial rendering of the yellow dot or possibly no dot at all. During the detection we take a number of steps to try and recover the partial dots and use the error correction code to recover the data where the whole dot is lost. Figure 2 shows an example of partial and full rendering. The image has been increased in scale to make the dots easier to see, the yellow dots are rendered as shades of grey in order that they can seen on a printed version of this paper.

\section{DETECTION}

The detection process involves initial preprocessing comprising three steps: Cropping, Thresholding, and Erosion and Dilation. This is followed by the actual detection of the yellow dots. The rotation correction is handled during the detection of the dots using a process we term as Window Adjustment. 


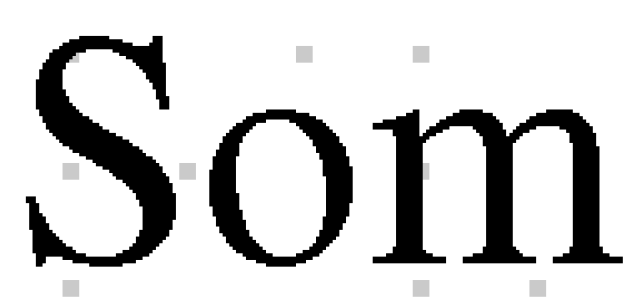

Figure 2: Example Dot Rendering

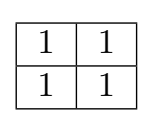

(a) Erosion

\begin{tabular}{|l|l|l|}
\hline 1 & 1 & 1 \\
\hline 1 & 1 & 1 \\
\hline 1 & 1 & 1 \\
\hline
\end{tabular}

(b) Dilation

Figure 3: Structuring Elements

\subsection{Cropping}

After scanning the document it is not unusual to have noise, or a yellowish colour, around the edges of the image. This is typically caused by a less than perfectly fitting scanner lid, or slight inaccuracies in the placement of the paper on the scanner glass. To counter this we initially crop 10 pixels from around the outside of the image. This is possible to do, without risking loss of dots, because we used a margin of 100 pixels around the page during the embedding process as defined in Section 3.

\subsection{Thresholding}

We next threshold the document to create a binary image, of what should be just yellow dots. This is to improve the performance and make further processing easier. We search for yellow pixels with colour within the following range:

$$
\begin{aligned}
& 215<R \leq 255 \\
& 215<G \leq 255 \\
& 100<B<200
\end{aligned}
$$

setting them as black pixels in the binary image and all others as white pixels. Ideally the binary image now only contains the yellow dots represented by black pixels, however, there is still the possibility that some noise has also been included. From this point on we will still refer to the yellow dots even though the pixels themselves are black. When discussing particular techniques that are used at a pixel level we will correctly refer to them as black pixels, even though they represent a yellow dot.

\subsection{Erosion and Dilation}

Following the threshold operation we perform an erosion using the structuring element in Figure 3a. This removes noise created during the printing and scanning process. A dilation operation subsequently follows using the structuring element in Figure 3b. This fills in any incomplete dots and increases the size of the partial dots created during the embedding.

\subsection{Locating the Grid and Dots}

The concept of detecting the dots is to use a moving window, sized to be equivalent to a single cell in the grid, and to evaluate the pixels within the window to determine whether it contains a yellow dot or not. The window is moved along the rows and columns to cover the entire grid. The exact movements are determined by calculating the size of the detected grid augmented with the values from the Window Adjustment process. 


\subsubsection{Locating the Grid}

The first step in locating the grid is to find the top left corner of it. This is achieved using horizontal and vertical profiles, discussed in more detail in, ${ }^{810}$ and. ${ }^{11}$ These provide a profile of the horizontal or vertical plane of an image. They allow us to find the top most and left most plane that has yellow dots in it. We use a threshold of 5 when creating the profile to avoid picking up any remaining noise. The combination of the horizontal and vertical profile gives us $W_{x}$ and $W_{y}$ as the top left location of the window as well as the $W_{w}$ and $W_{h}$ as the width and height. In effect this has given us an initial area for the top left cell of the grid.

\subsubsection{Window Adjustment}

The Window Adjustment process is used to correct the location of the moving window based on the where we have already found yellow dots. The process begins by searching within the initial window, calculated above, for the top left black pixel. If a black pixel is found, $\tilde{W}_{x}$ and $\tilde{W}_{y}$ are set to the relative distances between the pixel and $W_{x}$ and $W_{y}$ respectively, otherwise they are set to 0 .

We can now calculate a more accurate value for $W_{w}$ and $W_{h}$, which will be equivalent to the size of a single cell in the grid. The profiles used in Section 5.4.1 gives us the left, right, top and bottom most pixels in the document. This gives us the detected grid width and height, $D G_{w}$ and $D G_{h}$. Since we know how many rows and columns we have, 194 and 135 respectively, we can calculate the $W_{w}$ and $W_{h}$ as follows:

$$
\begin{aligned}
& W_{w}=D G_{w} / 135 \\
& W_{h}=D G_{h} / 194
\end{aligned}
$$

The starting location for the moving window is centred over any found black pixel, as follows:

$$
\begin{aligned}
& W_{x}=\left(W_{x}+\tilde{W}_{x}\right)-W_{w} / 2 \\
& W_{y}=\left(W_{y}+\tilde{W}_{y}\right)-W_{h} / 2
\end{aligned}
$$

\subsubsection{Moving the Window}

We now iterate through each row and column within the grid. The moving window is set as follows:

$$
\begin{aligned}
& W_{x}=\left(i \times W_{w}\right)+\tilde{W}_{x} \\
& W_{y}=\left(j \times W_{h}\right)+\tilde{W}_{y}
\end{aligned}
$$

where $i$ and $j$ refer to the current row and column in which we are looking. If a black pixel is found within the window, then a ' 1 ' bit is added to the data buffer. If no black pixels are found a ' 0 ' bit is added to the data buffer. In the case of a black pixel being found we then calculate the average location of all the black pixels found in the window to give us $D_{x}$ and $D_{y} . D_{x}$ and $D_{y}$ are relative to the whole image. We then make the following adjustments:

$$
\begin{aligned}
& \tilde{W}_{x}=\tilde{W}_{x}+\left(D_{x}-W_{x}\right)+\frac{W_{w}}{2} \\
& \tilde{W}_{y}=\tilde{W}_{y}+\left(D_{y}-W_{y}\right)+\frac{W_{h}}{2}
\end{aligned}
$$

If we are looking within the left most column the adjustments made to $\tilde{W}_{x}$ and $\tilde{W}_{y}$ are permanent. Otherwise the adjustment is only valid within the row it is calculated. At the end of each row the $\tilde{W}_{x}$ and $\tilde{W}_{y}$ are reset to the values they were at the start of the row. At the end of this process the data has been recovered and can be passed to the error correction code to process. 
Table 1: Turbo codes with block size $N=26190$

\begin{tabular}{c|cc|cc|c}
\hline & $K$ & $S$ & Feedback & Output & Puncturing \\
\hline $\mathrm{A}$ & 13095 & 45 & 37 & 33 & Odd/Even \\
$\mathrm{B}$ & 8730 & 37 & 37 & 33 & None \\
$\mathrm{C}$ & 5238 & 30 & 37 & 33,25 & None \\
\hline
\end{tabular}

\section{ERROR-CORRECTING}

The embedding and detection process cannot be expected to be an error free undertaking. During embedding, there will be occasions when it is not possible to embed the dot in a particular cell due to lack of whitespace. Likewise there are likely to be occasions when dots are lost due to distortion in the print and scan process or imperfections in the scanning process. We counter these problems using Error-Correcting Codes (ECC) of large block size and various code rates.

For simplicity in this proof-of-concept, we restrict ourselves to binary codes, under the assumption that the complete embedding $\rightarrow$ print $\rightarrow$ scan $\rightarrow$ detection process behaves like a Binary Symmetric Channel (BSC). Given that we are expecting channel error rates of around $7 \%$ or more, we restrict ourselves to code rates of $\frac{1}{2}$ or less. Turbo codes, originally presented by Berrou et al., ${ }^{12}$ are widely regarded as ideal codes for low rate applications with large block sizes, and therefore eminently suited here.

For the required block size $N=26190(135 \times 194)$, we design turbo codes of rates $\frac{1}{2}, \frac{1}{3}$, and $\frac{1}{5}$, as tabulated in Table 1. For each code, we show respectively the message size $K$ (equal to the interleaver size), and the spread $S$ for the $S$-random interleaver used. ${ }^{13}$ The feedback and output polynomials are shown in the usual octal notation. Codes $B$ and $C$ achieve the required overall code rate without puncturing, while Code $A$ is based on Code $B$ with odd/even puncturing to achieve a code rate of $\frac{1}{2}$.

The codes are decoded using 20 iterations of the MAP algorithm. The decoder input probabilities are calculated assuming a BSC with an arbitrary cross-over rate $p$.

\section{EXPERIMENTATION}

We created five sample documents, a cartoon, two examples of architectural plans, a page of text and a letter. We initially embedded dots of $5 \times 5$ using the different error correcting codes and successfully detected them without error in all but one case as shown in Table 2. Consequently, we experimented with reducing the size of the dots to $3 \times 3$. The documents were printed at 600 dpi on a Hewlett Packard F2180 and were scanned using an Epson V700 Photo at 600 dpi and 48 bit colour. The print/scan experiment was performed once for each document. The cartoon document, with message of size of 8730 , was the only document at $5 \times 5$ that caused difficulties. The document was a greyscale instead of binary and contained a large box surrounding the cartoon. During scanning the greyscale areas would often be detected with yellow pixels in them. This caused noise and inturn synchronisation problems.

We successfully recovered the message, when embedding with a dot size of $3 \times 3$, in a third of all cases. The smaller dot size caused an increase in errors due to the erosion process, described in Section 5.3, removing both the noise and partial yellow dots.

\section{CONCLUSION AND ONGOING WORK}

We have demonstrated a proof-of-concept for automatically embedding and detecting yellow printer dots in binary documents. The experimental results have shown the potential of the scheme to flawlessly recover a message after printing and scanning, for different types of document. The current technique has significant scope for further improvement, including optimising dot size, cell size as well as improvements in the demodulation technique. We are currently undertaking some of this further work.

Currently our scheme focusses on binary documents, since colour could potentially conflict with the yellow dots and cause excessive errors. There is potential to use it for greyscale images as well, although they would need to be line art or images that still contained a significant proportion of white background. The reason for this is that we do not wish to embed in non-white background areas since this will increase the perceptibility. 
Table 2: Experiment Results

\begin{tabular}{r|c|c|c}
\hline & Code & $\begin{array}{c}\text { Bit Errors } \\
\text { at } 5 \times 5\end{array}$ & $\begin{array}{c}\text { Bit Errors } \\
\text { at } 3 \times 3\end{array}$ \\
\hline Cartoon & A & 0 & 3089 \\
Plan 1 & A & 0 & 2518 \\
Plan 2 & A & 0 & 2504 \\
Text & A & 0 & 2646 \\
Letter & A & 0 & 1439 \\
\hline Cartoon & B & 46 & 1289 \\
Plan 1 & B & 0 & 4 \\
Plan 2 & B & 0 & 771 \\
Text & B & 0 & 744 \\
Letter & B & 0 & 2429 \\
\hline Cartoon & C & 0 & 0 \\
Plan 1 & C & 0 & 0 \\
Plan 2 & C & 0 & 0 \\
Text & C & 0 & 0 \\
Letter & C & 0 & 0 \\
\hline
\end{tabular}

\section{REFERENCES}

[1] et al, S. H. L., "Document identification for copyright protection using centroid detection," IEEE Transactions on Communication 46(3), 372-383 (1998).

[2] Tuohey, J., "Government uses color laser printer technology to track documents." Internet http://www . pcworld.com (November 2004). last accessed 22/05/2009.

[3] Foundation, E. F., "Is your printer spying on you." Internet. http://www .eff.org/issues/printers last accessed 22 May 2009.

[4] de Vries, W., "Dutch track counterfeits via printer serial numbers." Internet http://www.pcworld.com (October 2004). last accessed 22/05/2009.

[5] S., H. and F., F., "Tracking codes in photocopiers and colour laser printers." Internet (March 2008). http: //www. europarl. europa.eu/sides/getDoc.do?reference=E-2007-5724\&language=EN last accessed 22 May 2009.

[6] Foundation, E. F., "Docucolor tracking dot decoding guide." Internet. http://w2.eff.org/Privacy/ printers/docucolor/ last accessed 22 May 2009.

[7] Villán, R., Voloshynovskiy, S., Koval, O., Deguillaume, F., and Pun, T., "Tamper-proofing of electronic and printed text documents via robust hashing and data-hiding," in [Proceedings of the SPIE], 6505 (Feb. 2007).

[8] Zou, D. and Shi, Y. Q., "Formatted text document data hiding robust to printing, copying and scanning," in [IEEE International Symposium on Circuits and Systems (ISCAS05)], (May 2005).

[9] Culnane, C., Treharne, H., and Ho, A. T. S., "Authenticating binary text documents using a localising omac watermark robust to printing and scanning," in [International Workshop on Digital Watermarking (IWDW07)], 177-191, LNCS 5041 (2007).

[10] Low, S. H. and Maxemchuk, N. F., "Performance comparison of two text marking methods," IEEE Journal on Special Areas in Communications 16(4), 561-572 (1998).

[11] Culnane, C., Treharne, H., and Ho, A. T. S., "A new multi-set modulation technique for increasing hiding capacity of binary watermark for print and scan processes," in [International Workshop on Digital Watermarking (IWDW06)], 96-110, LNCS 4285 (2006).

[12] Berrou, C., Glavieux, A., and Thitimajshima, P., "Near Shannon limit error-correcting coding and decoding: Turbo-codes," in [Proceedings of the IEEE International Conference on Communications], 1064-1070 (May 1993).

[13] Divsalar, D. and Pollara, F., "Turbo codes for deep-space communications," TDA progress report 42-120, Jet Propulsion Laboratory, California Institute of Technology (Feb. 15th, 1995). 